\title{
Reliability of plantar pressure platforms
}

\author{
Jocelyn F. Hafer ${ }^{a,}{ }^{\star}$, Mark W. Lenhoff ${ }^{a}$, Jinsup Song ${ }^{b}$, Joanne M. Jordan ${ }^{c}$, Marian T. \\ Hannan ${ }^{\mathrm{d}, \mathrm{e}}$, and Howard J. Hillstrom ${ }^{\mathrm{a}}$ \\ aHospital for Special Surgery, New York, NY, USA \\ bTemple University School of Podiatric Medicine, Philadelphia, PA, USA \\ cUniversity of North Carolina, Chapel Hill, NC, USA \\ dHarvard Medical School, Boston, MA, USA \\ eInstitute for Aging Research, Hebrew SeniorLife, Boston, MA, USA
}

\begin{abstract}
Plantar pressure measurement is common practice in many research and clinical protocols. While the accuracy of some plantar pressure measuring devices and methods for ensuring consistency in data collection on plantar pressure measuring devices have been reported, the reliability of different devices when testing the same individuals is not known. This study calculated intra-mat, intra-manufacturer, and inter-manufacturer reliability of plantar pressure parameters as well as the number of plantar pressure trials needed to reach a stable estimate of the mean for an individual. Twenty-two healthy adults completed ten walking trials across each of two Novel emed-x® and two Tekscan MatScan ${ }^{\circledR}$ plantar pressure measuring devices in a single visit. Intraclass correlation (ICC) was used to describe the agreement between values measured by different devices. All intraplatform reliability correlations were greater than 0.70 . All inter-emed- $x \circledR$ reliability correlations were greater than 0.70 . Inter-emed- $x \circledR$ reliability correlations were greater than 0.70 in 31 and 52 of 56 parameters when looking at a 10-trial average and a 5-trial average, respectively. Intermanufacturer reliability including all four devices was greater than 0.70 for 52 and 56 of 56 parameters when looking at a 10-trial average and a 5-trial average, respectively. All parameters reached a value within $90 \%$ of an unbiased estimate of the mean within five trials. Overall, reliability results are encouraging for investigators and clinicians who may have plantar pressure data sets that include data collected on different devices.
\end{abstract}

\section{Keywords}

Plantar pressures; Reliability; Pressure measurement; Gait

\footnotetext{
(C) 2013 Elsevier B.V. All rights reserved.

* Corresponding author at: 535 E. 70th Street, New York, NY 10021, USA. Tel.: +1 212606 1215. haferj@hss.edu, jocelynfhafer@gmail.com.

Study information This study was approved by the Institutional Review Board at the Hospital for Special Surgery.

Conflict of interest statement We, the authors, affirm that we have no financial affiliation (including research funding) or involvement with any commercial organization that has a direct financial interest in any matter included in this manuscript, except as disclosed in an attachment and cited in the manuscript.
}

Appendix A. Supplementary data Supplementary data associated with this article can be found, in the online version, at http:// dx.doi.org/10.1016/j.gaitpost.2013.01.028. 


\section{Introduction}

Plantar pressure measurements are part of many clinical and research protocols. Several companies manufacture plantar pressure platforms, and accuracy and reliability of individual makes of platforms have been demonstrated [1-3]. However, reliability (i.e. reproducibility of an individual's plantar pressure parameters) between platforms of different manufacturers or platforms of the same manufacturer has not been established. Platform technology is manufacturer-specific which results in different resolutions, sensor types, sampling rates, and ranges of detectable pressure [4]. These differences may cause plantar pressure measurements of an individual to vary from one manufacturer's platform to another's. Researchers and clinicians may wish to compare data collected on platforms of different manufacturers due to changes in available equipment over time or to combine data sets from separate studies or clinics. Therefore, the primary purpose of this study is to determine the reliability between and within Novel emed- $x{ }^{\circledR}$ and Tekscan MatScan ${ }^{\circledR}$ plantar pressure platforms.

Additionally, the minimum number of trials required for an unbiased estimate of the mean for plantar pressures is not established. A previous study reported coefficients of reliability for whole-foot plantar pressure measurements based on the number of trials collected [2]. As a secondary purpose, this study will report parameter stability for ten regions of the foot for the emed-x® and MatScan ${ }^{\circledR}$ to determine an appropriate number of data collection trials.

\section{Methods}

All methods were approved by the IRB and subjects completed informed consent forms.

\subsection{Subjects}

Twenty-two participants were recruited. Participants were healthy adults aged $28.6 \pm 9.9$ years (range: $20-53$ ), height $1.7 \pm 0.1 \mathrm{~m}$, and mass $75.5 \pm 13.4 \mathrm{~kg}$. Thirteen participants were male. No participants had a history of gait abnormality. Resting calcaneal stance position was recorded for each subject to describe foot alignment. On average, subjects were in $6.2 \pm 5.5^{\circ}$ of calcaneal valgus, indicating normal foot alignment [5].

\subsection{Platforms}

Plantar pressure measures were recorded on four platforms, two emed-x® plates (Novel, Munich, Germany) and two MatScans ${ }^{\circledR}$ (Tekscan, Boston, MA). The emed-x® consists of 6080 capacitance-based force transducers at a resolution of 4 sensors $/ \mathrm{cm}^{2}$. The MatScan ${ }^{\circledR}$ consists of 2288 resistive sensors at a resolution of 1.4 sensors $/ \mathrm{cm}^{2}$.

All platforms were within one year of being calibrated to manufacturers' standards.

\subsection{Procedure}

Participants completed 10 satisfactory walking trials on each of the 4 platforms. Only rightfoot data were collected using the two-step method to expedite data collection. The two-step method consists of the participant landing on the pressure platform on the second step. This method has been shown to be consistent with midgait methods [6]. Participants were instructed to look straight ahead to deter targeting and velocity was monitored using stopwatches to ensure consistency. Trials varying $\geq 10 \%$ in time were considered acceptable.

\subsection{Data analysis}

Novel Projects software (Novel, Munich, Germany) was used to analyze plantar pressure data using a standard twelve-region mask. The accuracy of automasking using this software 
has been previously shown [7]. The following plantar pressure parameters were calculated: peak pressure $\left(\mathrm{N} / \mathrm{cm}^{2}\right)$, maximum force $(\mathrm{N})$, area $\left(\mathrm{cm}^{2}\right)$, pressure-time integral $\left(\mathrm{N} \mathrm{s} / \mathrm{cm}^{2}\right)$, and force-time integral ( $\mathrm{N} \mathrm{s}$ ).

These parameters were calculated from the maximum pressure throughout stance phase plot for the entire foot contact region (total) and the following 10 regions of the foot:

1. Hallux

2. Metatarsal head one (MH1)

3. Metatarsal head two (MH2)

4. Metatarsal head three (MH3)

5. Metatarsal head four (MH4)

6. Metatarsal head five (MH5)

7. Medial arch

8. Lateral arch

9. Medial heel

10. Lateral heel

In addition, center of pressure excursion index (CPEI) [8] was calculated. CPEI is a measure of dynamic pronation. A total of 56 parameters were calculated.

Parameter stability was determined by calculating the cumulative average (average of first trial, first 2 trials, first 3 trials... average of 10 trials) of each parameter for each subject and identifying the number of trials required for the average to be within $90 \%$ of the unbiased estimate (10-trial mean). A custom MatLab script was used to normalize all cumulative averages to percent of the 10-trial mean to allow for comparisons across subjects.

\subsection{Statistics}

Descriptive statistics were calculated for all measures to determine whole-group average and standard deviation for each plantar pressure parameter in each masked region, in the total foot, and for CPEI for all platforms. Analyses were done using SPSS v19 (IBM, Chicago, IL). Reliability coefficients were calculated for all parameters and all foot regions as described below.

2.5.1. Intra-platform reliability-To determine the reliability between measures taken with the same platform, intraclass correlation (ICC) type $(2,1)$ was calculated. ICCs compared the average of the first five trials over all subjects with the average of the last five trials.

2.5.2. Inter-platform reliability-To determine the reliability between measures taken with different platforms, inter-platform reliability was calculated between platforms of the same manufacturer (emed- $x \circledR 1$ to emed- $x \circledR 2$ and MatScan ${ }^{\circledR} 1$ to MatScan® ${ }^{\circledR}$ ) and between platforms of different manufacturers (emed- $x \circledR 1$ to emed- $x{ }^{\circledR} 2$ to MatScan ${ }^{\circledR} 1$ to MatScan ${ }^{\circledR}$ 2). ICCs of type $(2,1)$ were calculated for all comparisons using both the average of five trials and the average of 10 trials. 


\section{Results}

\subsection{Intra-platform reliability (Table 1)}

51 of 56 ICC values for intra-platform reliability were greater than or equal to 0.90 .

\subsection{Inter-platform reliability (Table 2)}

3.2.1. 10-trial average-Inter-emed- $x ®$ reliability was greater than 0.70 for all parameters. Inter-MatScan ${ }^{\circledR}$ reliability was greater than 0.70 for 31 of 56 parameters. Intermanufacturer reliability was greater than 0.70 for 52 of 56 parameters.

3.2.2. 5-Trial average-Inter-emed- $x ®$ reliability was greater than 0.70 for all parameters. Inter-MatScan ${ }^{\circledR}$ reliability was greater than or equal to 0.70 for 52 of 56 parameters.

Inter-manufacturer reliability including all four platforms was greater than 0.70 for all parameters.

\subsection{Parameter stability (Fig. 1)}

All parameters reached less than $10 \%$ different from the unbiased estimate of the mean within 5 trials. Several parameters reached this threshold at the first trial including peak pressure in Total, Medial and Lateral Heel, and MH2 regions; maximum force in Total, Medial and Lateral Heel, MH2 and MH3 regions; pressure-time integral in Total, MH2, and MH3 regions, force-time integral in Total, MH2, and MH3 regions; and area in Total, Medial and Lateral Heel, MH2, MH3, and MH4 regions.

\section{Discussion}

Overall, reliability results are consistent for this asymptomatic healthy group of subjects.

Intra-platform reliability results are encouraging, especially since many studies involve data collected on a single platform. These results indicate that plantar pressure measurements are consistent when measured on the same emed- $x{ }^{\circledR}$ or MatScan ${ }^{\circledR}$. Previous studies have reported reliability of the MatScan ${ }^{\circledR}[3]$ and the emed-sf ${ }^{\circledR}[2]$ and have found similar moderate to high reliability. The results of the present study also indicate that, when collected on a single platform, plantar pressure data reach a stable estimate of a 10-trial mean within 5 trials. For most parameters, reliable data can be achieved with as few as 3 walking trials. Previous studies have found similar results for individual Novel platforms emed-sf® [4] and emed-F® [2].

Inter-platform reliability results indicate that data collected from the same subject on different plantar pressure platforms are consistent. Inter-emed-x®, inter-MatScan ${ }^{\circledR}$, and inter-manufacturer reliability exceeded a reliability coefficient of 0.70 on most plantar pressure values. This indicates that data collected on different platforms and even on different platforms from different manufacturers are equivalent.

\section{Supplementary Material}

Refer to Web version on PubMed Central for supplementary material.

\section{Acknowledgments}

This work was supported by NIAMS grant R01 AR047853. 


\section{References}

[1]. Giacomozzi C. Hardware performance assessment recommendations and tools for baropodometric sensor systems. Annali dellIstituto Superiore di Sanita. 2010; 46:158-67.

[2]. Hughes J, Pratt L, Linge K, Clark P, Klenerman L. Reliability of pressure measurements: the EMED F system. Clinical Biomechanics (Bristol Avon). 1991; 6:14-8.

[3]. Zammit GV, Menz HB, Munteanu SE. Reliability of the TekScan MatScan(R) system for the measurement of plantar forces and pressures during barefoot level walking in healthy adults. Journal of Foot and Ankle Research. 2010; 3:11. [PubMed: 20565812]

[4]. Giacomozzi C. Appropriateness of plantar pressure measurement devices: a comparative technical assessment. Gait and Posture. 2010; 32:141-4. [PubMed: 20399101]

[5]. Astrom M, Arvidson T. Alignment and joint motion in the normal foot. Journal of Orthopaedic and Sports Physical Therapy. 1995; 22:216-22. [PubMed: 8580949]

[6]. McPoil TG, Cornwall MW, Dupuis L, Cornwell M. Variability of plantar pressure data: a comparison of the two-step and midgait methods. Journal of the American Podiatric Medical Association. 1999; 89:495-501. [PubMed: 10546420]

[7]. Ellis SJ, Stoecklein H, Yu JC, Syrkin G, Hillstrom HJ, Deland JT. The accuracy of an automasking algorithm in plantar pressure measurements. HSS Journal. 2011; 7:57-63. [PubMed: 22294959]

[8]. Song J, Hillstrom HJ, Secord D, Levitt J. Foot type biomechanics. Comparison of planus and rectus foot types. Journal of the American Podiatric Medical Association. 1996; 86:16-23. [PubMed: 8808320] 

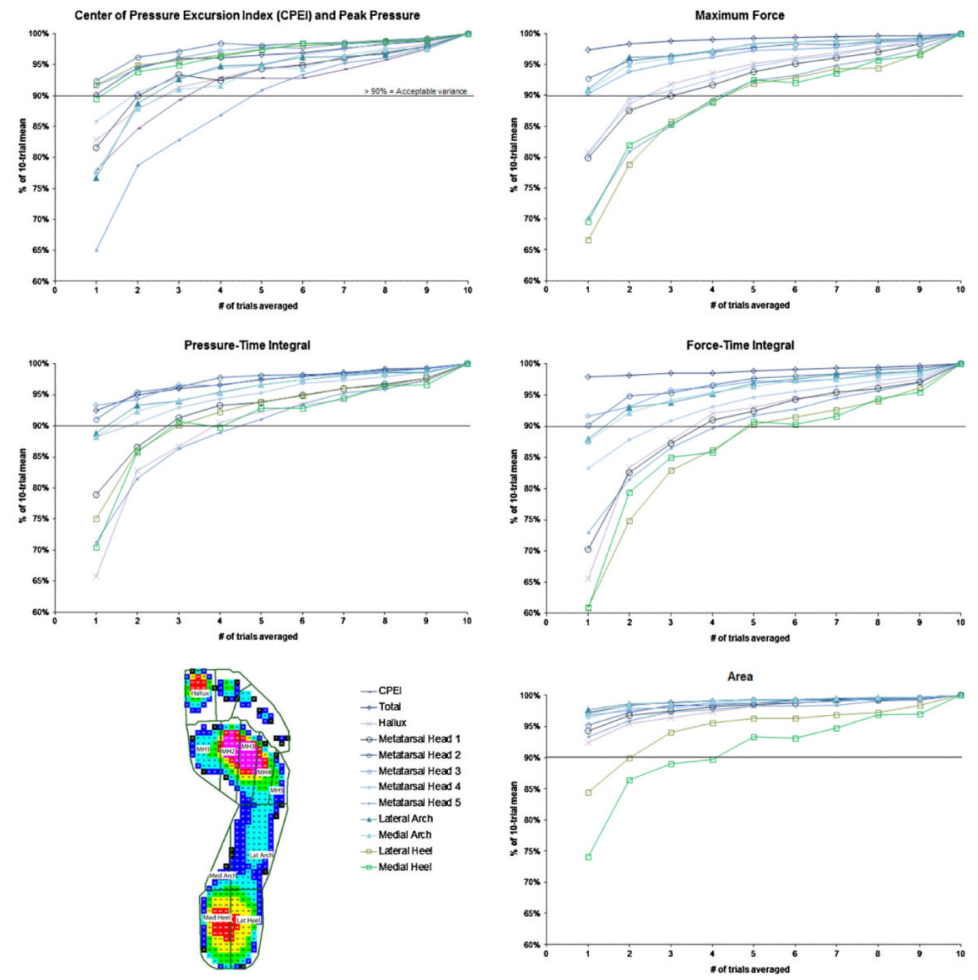

Fig. 1.

Parameter stability as $\%$ of the 10 -trial mean by number of trials averaged. Horizontal line at 90\% indicates acceptable threshold. All parameters reached this threshold within 5 trials. Key displays regions of the foot by label and where the regions are on an example plantar pressure trial. "Total" indicates whole foot. 
Table 1

ICC $(2,1)$ and standard error of the mean (SEM) values for intra-mat correlation. M1 and M2 are MatScan platforms, E1 and E2 are emed-x platforms. For each platform, the mean of the first 5 trials was compared to the mean of the last 5 trials. Bold values indicate $\mathrm{ICC}>0.90$.

\begin{tabular}{|c|c|c|c|c|c|c|c|c|}
\hline & \multicolumn{2}{|c|}{ M1 1-5 v 6-10 } & \multicolumn{2}{|c|}{ M2 1-5 v 6-10 } & \multicolumn{2}{|c|}{ E1 1-5 v 6-10 } & \multicolumn{2}{|c|}{ E2 1-5 v 6-10 } \\
\hline & ICC & SEM & ICC & SEM & ICC & SEM & ICC & SEM \\
\hline CPEI (\%) & 0.94 & 0.03 & 0.95 & 0.15 & 0.91 & 0.01 & 0.94 & 0.23 \\
\hline \multicolumn{9}{|c|}{ Peak pressure $\left(\mathrm{N} / \mathrm{cm}^{2}\right)$} \\
\hline Total & 0.97 & 0.00 & 0.98 & 0.04 & 0.99 & 0.05 & 0.99 & 0.12 \\
\hline Hallux & 0.98 & 0.12 & 0.94 & 0.13 & 0.98 & 0.06 & 0.97 & 0.00 \\
\hline MH1 & 0.96 & 0.00 & 0.83 & 0.10 & 0.93 & 0.15 & 0.98 & 0.06 \\
\hline MH2 & 0.99 & 0.00 & 1.00 & 0.04 & 1.00 & 0.00 & 1.00 & 0.00 \\
\hline MH3 & 0.98 & 0.05 & 0.99 & 0.01 & 0.99 & 0.00 & 0.99 & 0.01 \\
\hline MH4 & 0.95 & 0.13 & 0.95 & 0.10 & 0.93 & 0.02 & 0.95 & 0.10 \\
\hline MH5 & 0.95 & 0.15 & 0.88 & 0.13 & 0.93 & 0.02 & 0.93 & 0.34 \\
\hline Med arch & 0.99 & 0.01 & 0.97 & 0.01 & 0.99 & 000 & 0.96 & 0.07 \\
\hline Lat arch & 0.96 & 0.08 & 0.96 & 0.10 & 0.93 & 0.05 & 0.93 & 0.07 \\
\hline Med heel & 0.98 & 0.05 & 0.97 & 0.04 & 0.96 & 0.00 & 0.97 & 0.07 \\
\hline Lat heel & 0.98 & 0.03 & 0.98 & 0.03 & 0.97 & 0.02 & 0.98 & 0.00 \\
\hline \multicolumn{9}{|l|}{ Area $\left(\mathrm{cm}^{2}\right)$} \\
\hline Total & 1.00 & 0.02 & 0.99 & 0.05 & 0.99 & 0.02 & 0.99 & 0.01 \\
\hline Hallux & 0.93 & 0.00 & 0.97 & 0.03 & 0.99 & 0.00 & 0.98 & 0.00 \\
\hline MH1 & 0.91 & 0.01 & 0.90 & 0.03 & 0.99 & 0.01 & 0.97 & 0.01 \\
\hline MH2 & 0.95 & 0.00 & 0.96 & 0.00 & 0.99 & 0.00 & 0.99 & 0.00 \\
\hline MH3 & 0.95 & 0.02 & 0.97 & 0.00 & 0.94 & 0.03 & 0.98 & 0.00 \\
\hline MH4 & 0.98 & 0.02 & 0.96 & 0.03 & 0.98 & 0.01 & 0.96 & 0.02 \\
\hline MH5 & 0.96 & 0.01 & 0.93 & 0.00 & 0.98 & 0.00 & 0.95 & 0.00 \\
\hline Med arch & 0.96 & 0.04 & 0.95 & 0.02 & 0.99 & 0.00 & 0.94 & 0.02 \\
\hline Lat arch & 0.99 & 0.00 & 0.97 & 0.07 & 0.97 & 0.01 & 0.97 & 0.02 \\
\hline Med heel & 0.98 & 0.02 & 0.98 & 0.01 & 0.99 & 0.00 & 0.99 & 0.00 \\
\hline Lat heel & 0.98 & 0.01 & 0.98 & 0.01 & 1.00 & 0.00 & 0.99 & 0.00 \\
\hline \multicolumn{9}{|c|}{ Maximum force $(\mathrm{N})$} \\
\hline Total & 0.99 & 0.10 & 1.00 & 0.33 & 1.00 & 0.11 & 1.00 & 0.09 \\
\hline Hallux & 0.97 & 0.54 & 0.97 & 0.85 & 0.98 & 0.28 & 0.96 & 0.20 \\
\hline MH1 & 0.94 & 0.11 & 0.90 & 1.25 & 0.93 & 0.22 & 0.97 & 0.80 \\
\hline MH2 & 0.97 & 0.29 & 0.99 & 0.25 & 0.99 & 0.03 & 0.99 & 0.03 \\
\hline MH3 & 0.96 & 0.52 & 0.96 & 0.65 & 0.97 & 0.36 & 0.97 & 0.25 \\
\hline MH4 & 0.94 & 0.35 & 0.91 & 1.23 & 0.91 & 0.21 & 0.92 & 0.84 \\
\hline MH5 & 0.94 & 0.26 & 0.91 & 0.36 & 0.91 & 0.08 & 0.91 & 0.73 \\
\hline Med arch & 0.98 & 0.19 & 0.95 & 0.03 & 0.98 & 0.08 & 0.98 & 0.05 \\
\hline Lat arch & 0.98 & 0.37 & 0.98 & 0.30 & 0.97 & 0.11 & 0.96 & 0.74 \\
\hline Med heel & 0.97 & 1.03 & 0.97 & 0.20 & 0.99 & 0.06 & 0.96 & 1.18 \\
\hline
\end{tabular}




\begin{tabular}{|c|c|c|c|c|c|c|c|c|}
\hline & \multicolumn{2}{|c|}{ M1 1-5 v 6-10 } & \multicolumn{2}{|c|}{ M2 1-5 v 6-10 } & \multicolumn{2}{|c|}{$\underline{E 11-5 \text { v 6-10 }}$} & \multicolumn{2}{|c|}{ E2 1-5 v 6-10 } \\
\hline & ICC & SEM & ICC & SEM & ICC & SEM & ICC & SEM \\
\hline Lat heel & 0.94 & 0.63 & 0.97 & 0.19 & 0.98 & 0.01 & 0.97 & 0.44 \\
\hline \multicolumn{9}{|c|}{ Pressure-time integral $\left(\mathrm{N} \mathrm{s} / \mathrm{cm}^{2}\right)$} \\
\hline Total & 0.96 & 0.02 & 0.97 & 0.07 & 0.98 & 0.02 & 0.98 & 0.03 \\
\hline Hallux & 0.98 & 0.01 & 0.90 & 0.11 & 0.95 & 0.04 & 0.91 & 0.00 \\
\hline MH1 & 0.94 & 0.01 & 0.78 & 0.10 & 0.92 & 0.03 & 0.96 & 0.03 \\
\hline MH2 & 0.99 & 0.00 & 0.99 & 0.02 & 1.00 & 0.00 & 1.00 & 0.01 \\
\hline MH3 & 0.98 & 0.00 & 0.98 & 0.01 & 0.99 & 0.01 & 0.99 & 0.03 \\
\hline MH4 & 0.96 & 0.01 & 0.93 & 0.04 & 0.93 & 0.01 & 0.91 & 0.07 \\
\hline MH5 & 0.95 & 0.03 & 0.89 & 0.03 & 0.90 & 0.03 & 0.86 & 0.10 \\
\hline Med arch & 0.99 & 0.00 & 0.98 & 0.01 & 0.99 & 0.00 & 0.98 & 0.01 \\
\hline Lat arch & 0.96 & 0.02 & 0.97 & 0.02 & 0.95 & 0.02 & 0.95 & 0.03 \\
\hline Med heel & 0.94 & 0.02 & 0.95 & 0.00 & 0.96 & 0.01 & 0.89 & 0.02 \\
\hline Lat heel & 0.94 & 0.01 & 0.95 & 0.01 & 0.97 & 0.00 & 0.91 & 0.00 \\
\hline \multicolumn{9}{|c|}{ Force-time integral ( $\mathrm{N} \mathrm{s})$} \\
\hline Total & 0.99 & 0.01 & 0.99 & 0.18 & 1.00 & 0.04 & 1.00 & 0.26 \\
\hline Hallux & 0.97 & 0.08 & 0.94 & 0.39 & 0.96 & 0.03 & 0.92 & 0.12 \\
\hline MH1 & 0.92 & 0.10 & 0.85 & 0.53 & 0.91 & 0.05 & 0.92 & 0.22 \\
\hline MH2 & 0.98 & 0.07 & 0.99 & 0.05 & 0.99 & 0.01 & 0.99 & 0.05 \\
\hline MH3 & 0.97 & 0.13 & 0.96 & 0.01 & 0.97 & 0.09 & 0.96 & 0.24 \\
\hline MH4 & 0.96 & 0.04 & 0.93 & 0.16 & 0.91 & 0.00 & 0.90 & 0.35 \\
\hline MH5 & 0.96 & 0.03 & 0.92 & 0.02 & 0.92 & 0.03 & 0.90 & 0.23 \\
\hline Med arch & 0.98 & 0.15 & 0.97 & 0.02 & 0.99 & 0.01 & 0.98 & 0.02 \\
\hline Lat arch & 0.97 & 0.20 & 0.99 & 0.04 & 0.98 & 0.14 & 0.97 & 0.19 \\
\hline Med heel & 0.94 & 0.36 & 0.95 & 0.03 & 0.95 & 0.05 & 0.92 & 0.10 \\
\hline Lat heel & 0.93 & 0.16 & 0.95 & 0.13 & 0.98 & 0.04 & 0.95 & 0.11 \\
\hline
\end{tabular}

Gait Posture. Author manuscript; available in PMC 2014 July 01. 


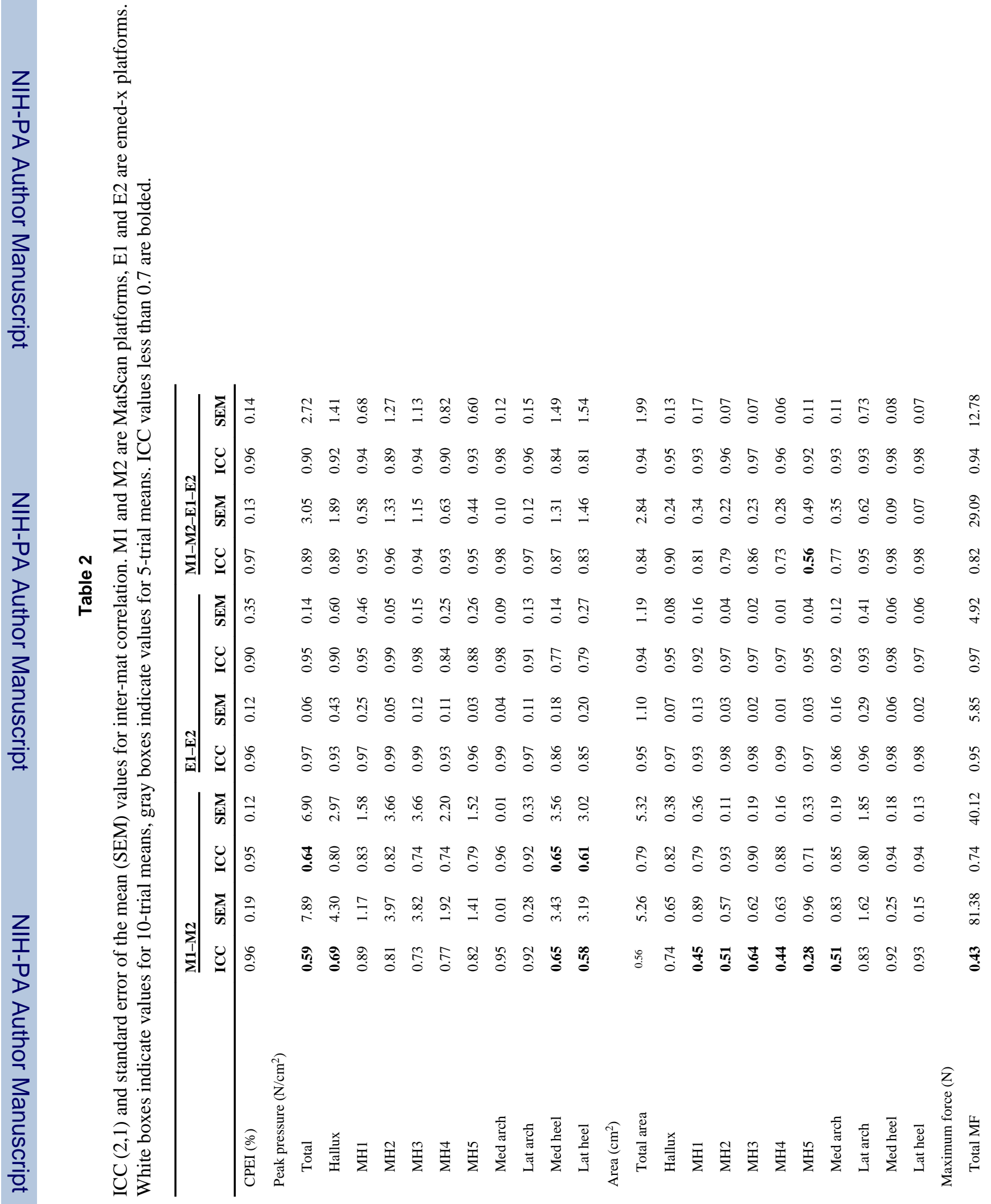




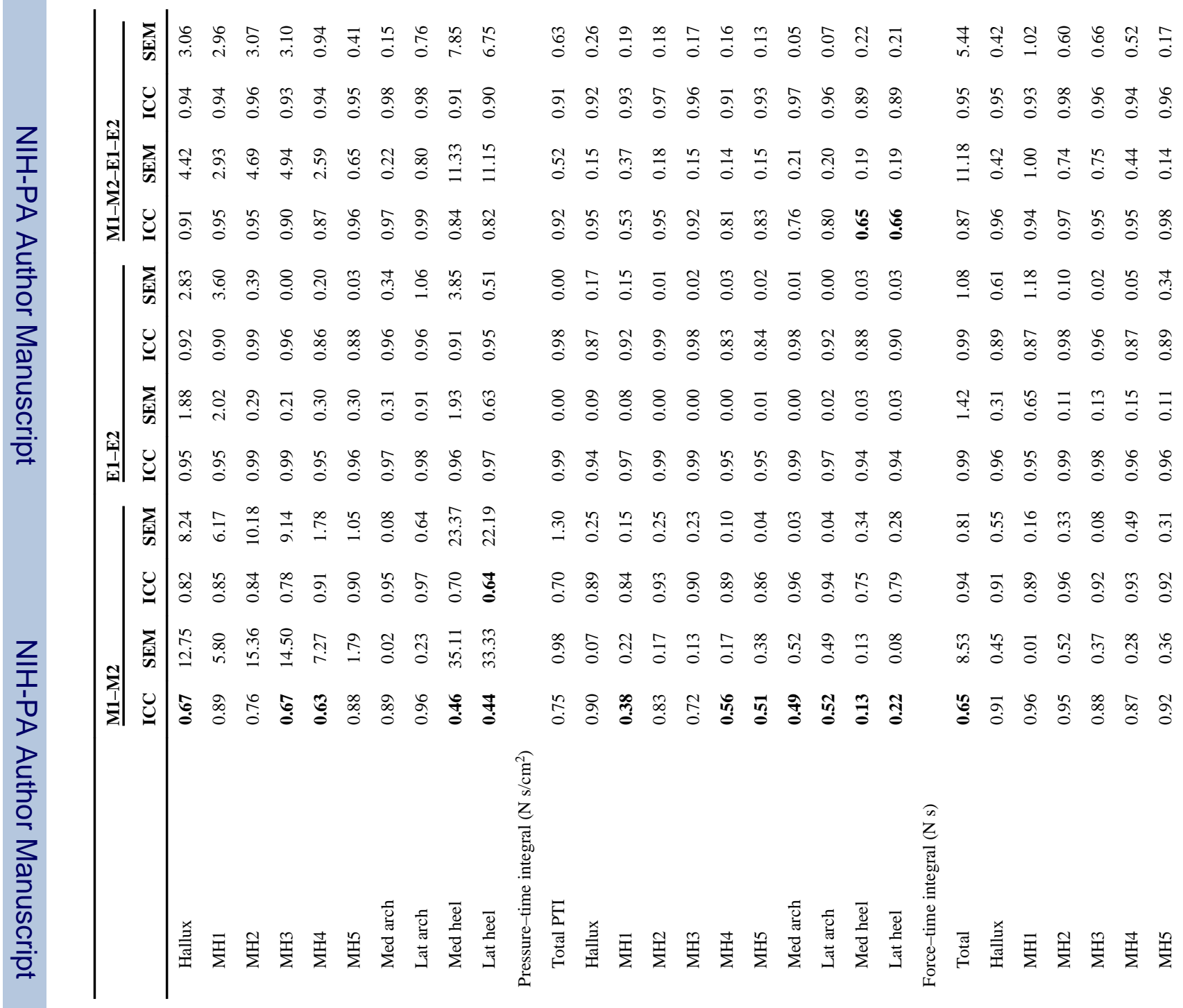




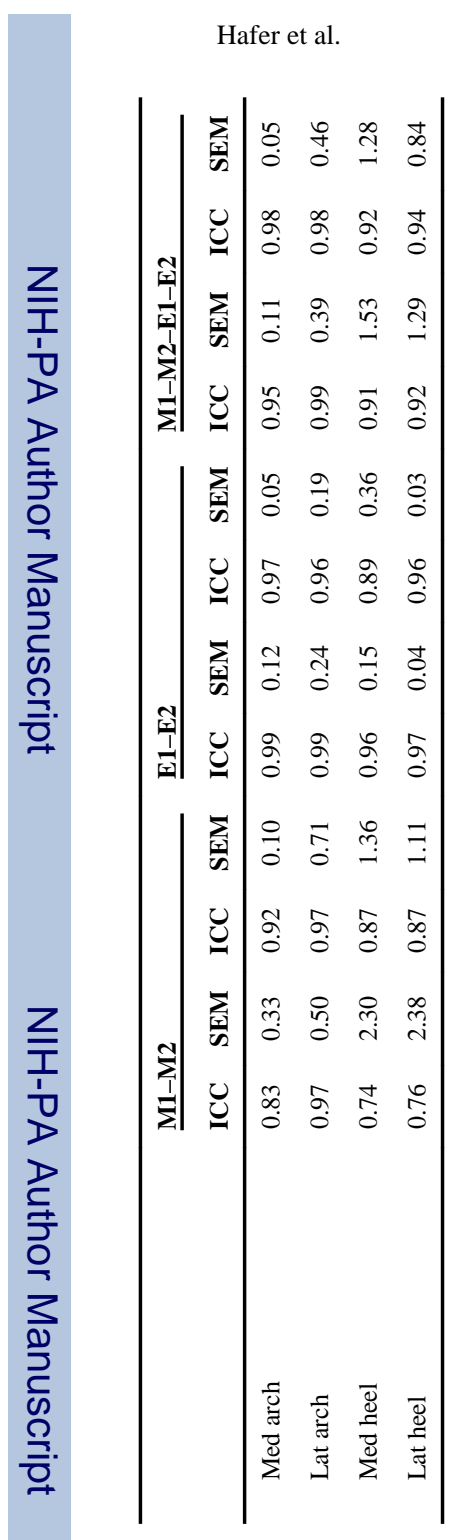

Gait Posture. Author manuscript; available in PMC 2014 July 01. 